\title{
Hybrid Control Method of Magnetically Controlled Shape Memory Alloy Actuator Based on Inverse Prandtl-Ishlinskii Model
}

\author{
Miaolei Zhou ${ }^{\dagger}$, Rui Xu*, Qi Zhang*, Ziying Wang* and Yu Zhao*
}

\begin{abstract}
The hysteresis nonlinearity of magnetically controlled shape memory alloy actuator is an obstacle in the achievement of high positioning accuracy. To eliminate the influence of hysteresis nonlinearity, a PID hybrid control method which uses the inverse Prandtl-Ishlinskii model as a feedforward controller is proposed in this paper. The PID parameters are searched by particle swarm optimization with cross and heredity function. The simulation results show that when the influence of external disturbance is not considered, the positioning accuracy of open loop control method is $0.67 \%$, and the positioning accuracy of PID hybrid control based on particle swarm optimization is $0.38 \%$; when the influence of external disturbance is considered, the positioning accuracy of open loop control method is $3.29 \%$, and the positioning accuracy of PID hybrid control based on particle swarm optimization is $1.65 \%$. This indicates that the proposed control method can effectively eliminate the impact of hysteresis nonlinearity and external disturbances on the positioning accuracy.
\end{abstract}

Keywords: Magnetically controlled shape memory alloy, Hysteresis nonlinearity, Prandtl-Ishlinskii model, Particle swarm optimization

\section{Introduction}

At the end of the 20th century, Vasilevf discovered a new functional material, magnetically controlled shape memory alloy (MSMA), which can produce deformation under the influence of the magnetic field and restore original shape when the magnetic field is removed [1-3]. The later researchers have concluded that this new functional material has advantages of the large output displacement and fast deformation frequency [4-5]. These merits make this new functional material become an excellent material in the field of micro/nano-driven [6-7]. But, the hysteresis nonlinearity reduces the control precision of the MSMA actuator and restricts its application and promotion. To broaden the applications of the MSMA actuator, it is crucial to eliminate the hysteresis nonlinearity of the MSMA actuator.

To achieve the high-precision positioning accuracy of smart materials, some control methods that combine the inverse model of hysteresis with a series of control methods are proposed. Chen Y. [8] designed a feedback controller based on the inverse modified Prandtl-Ishlinskii (PI) model to compensate the hysteresis. The positioning error of the piezoelectric actuator is reduced to less than $0.15 \mu \mathrm{m}$. Riccardi L. [9] developed a closed loop control law based on a modified Prandtl-Ishlinskii inverse model to decrease the error and verified the availability of this

$\dagger$ Corresponding Author: Dept. of Control Science and Engineering, Jilin University, China. (zml@jlu.edu.cn)

* Dept. of Control Science and Engineering, Jilin University, China. (\{ruixu14, zqi12, ziying11, zhaoyu13\}@mails.jlu.edu.cn)

Received: July 25, 2014; Accepted: April 1, 2016 proposed hybrid control method by the experiments on a micrometric positioning system based on the MSMA actuator. Zong X. [10] proposed a hybrid control method which combines an inverse preisach model with an adaptive sliding model controller to decrease the hysteresis nonlinearity of piezoelectric ceramic and proved that this controller can obtain good control accuracy. Ounissi A. [11] utilized the Coleman-Hodgdon hysteresis model as a dynamic model of piezoelectric actuators and used a classical proportion integration differentiation (PID) controller in order to achieve a tracking control of the nano-positioning system. The hysteresis model is identified by the particle swarm optimization (PSO) technique and the tracking error of this system is less than $100 \mathrm{~nm}$ by the proposed hybrid control method. Wang G. [12] proposed a hybrid control method with an asymmetric PrandtlIshlinskii hysteresis model and PID feedback control. By a series of experiment, it can be seen that this proposed hybrid control law is effective to decrease the hysteresis nonlinearity of piezoelectric actuator. Riccardi L. [13] designed an adaptive hysteresis compensation based on Krasnosel'skii - Pokrovskii model and a Proportion Integration (PI) controller in the feedback loop to eliminate the hysteresis phenomenon of the MSMA actuator under different temperature conditions. Finally, the experimental results prove that this proposed control method can effectively reduce the adverse effects caused by the temperature. Gu G. [14] adopted a hybrid control method which combines a feedforward controller based on an ellipse-based mathematic model with a PID feedback controller to eliminate the hysteresis characteristic of the piezoelectric actuator. The simulation and experimental 
results verify that the hysteresis error was reduced by up to $88 \%$. Sayyaadi H. [15] developed a hybrid control method which consists of the inverse generalized PI model in the feedforward controller and a PI feedback controller to eliminate the positioning error and the hysteresis nonlinearity of the shape memory alloy (SMA) actuator. By experiment and research, it can prove that the proposed control method has advantage in reducing the hysteresis nonlinearity and improving the positioning control accuracy of the SMA actuator.

In this paper, the hysteresis characteristic of the MSMA actuator is established by the PI model which is capable of describing the controlled object accurately, and the inverse PI model is identified by recursive weighted least-squares algorithm (RWLS). A hybrid control method is proposed to obtain a high positioning accuracy for the MSMA actuator. The method combines the inverse PI model in feedforward controller with a PID feedback controller whose parameters are adjusted by the PSO with cross and heredity function that can search the global optimum and can help the particle swarm jump out of the local optimum.

\section{Modeling and Controller Design of the MSMA Actuator}

\subsection{Modeling of the PI Model}

The PI model which utilizes the weighted superposition of a series of basic operator to describe the hysteresis nonlinearity inherits the modeling approach of the Preisach model [16-17]. The structure of the PI model is shown in Fig. 1. Its basic hysteresis operator (play operator) can be described as

$$
\begin{aligned}
& y_{r_{i}}(t)=p_{r_{i}}[x](t)= \\
& \left\{\begin{array}{lr}
x(t) & p_{r_{i}}[x](t-1)>x(t) \\
p_{r_{i}}[x](t-1) & p_{r_{i}}[x](t-1) \leq x(t) \leq p_{r_{i}}[x](t-1)+r_{i} \\
x(t)-r_{i} & p_{r_{i}}[x](t-1)+r_{i}<x(t)
\end{array}\right.
\end{aligned}
$$

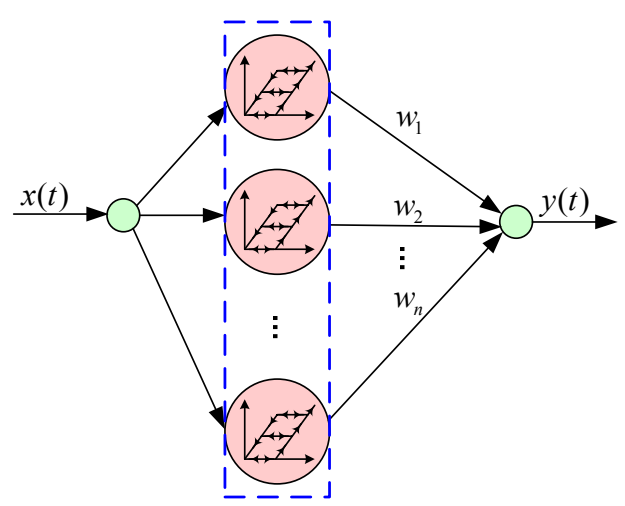

Fig. 1. Structure of PI model

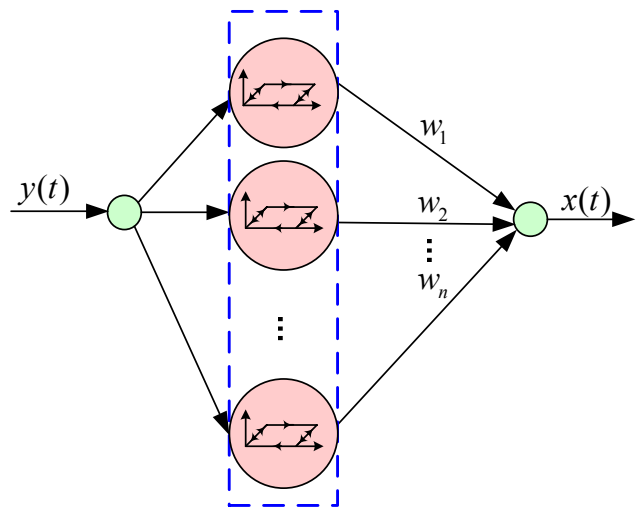

Fig. 2. Structure of the inverse PI model

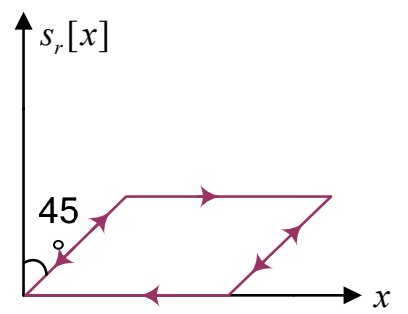

Fig. 3. Stop operator of the PI model

$$
y(t)=\sum_{i}^{n} w_{i} p_{r_{i}}[x(t)]
$$

where $x(t)$ and $y_{r_{i}}(t)$ are the input value and output value of the operator respectively, $r_{i}$ is the threshold value of the operator, $p_{r_{i}}[x](t)$ is the linear play operator, $y(t)$ is the output value of the model, $n$ is the amount of the operators, $w_{i}$ is the weighted vector.

\subsection{Inverse PI model of the MSMA actuator}

The inverse PI model which is based on the weighted superposition of a series of basic hysteresis operator is similar to the PI model, and the only difference is its basic hysteresis operator [18]. The structure of the inverse PI model is shown in Fig. 2.

One of advantages of the PI model is that it is easy to obtain its inverse model. The basic hysteresis operator (stop operator) of the inverse PI model is directly obtained by the basic hysteresis operator of the PI model (play operator). The solution equation is given in (3).

$$
s_{r}[x](t)=x(t)-p_{r}[x](t)
$$

where $s_{r}[x](t)$ is the stop operator, $x(t)$ is the input of the play operator.

The stop operator is described in Fig. 3.

\subsection{Identification method of the inverse PI model}

The amount of the stop operators used in the inverse PI 
model is much more than the amount of the play operators used in the PI model and it increases the computation quantity of the identification process. Therefore, RWLS [19-20] is utilized for identifying the weight parameters of the inverse model.

RWLS can be expressed by

$$
\begin{gathered}
\hat{\theta}(t)=\hat{\theta}(t-1)+L(t)\left[y(t)-\varphi^{T}(t) \hat{\theta}(t-1)\right] \\
L(t)=P(t-1) \varphi(t)\left[1+\varphi^{T}(t) P(t-1) \varphi(t)\right]^{-1} \\
P(t)=\left[I-L(t) \varphi^{T}(t)\right] P(t-1)
\end{gathered}
$$

where $\hat{\theta}$ is the parameters of the inverse PI model to be identified, $L(t)=P(t) \varphi(t), y(t)$ is the actual output signal, $\varphi(t)$ is the output of the stop operator, and $P(t)=\sum_{i=1}^{t} \varphi(i) \varphi^{T}(i)$.

By comparison of a series of simulation results, the number of stop operator is set to 2000 and the threshold of the $i$ th stop operator is selected by $r_{i}=\frac{i}{81} \max \left\{\|y(t)\|_{\infty}\right\}$ $i=1,2 \cdots 2000$.

\subsection{Controller design based on the inverse PI model}

\subsubsection{Open Loop Controller of the MSMA Actuator}

By the inverse control theory [21-23], the desired output signal which goes through the inverse model and the MSMA actuator is the equal of a unit mapping. In this section, an open loop controller is established by this theory. The structure of the open loop controller based on the inverse PI model is showed in Fig. 4, where $y_{d}$ is the desired output displacement, $u_{r}$ is the actual output of inverse model, $y$ is the actual output of the MSMA actuator, and $u_{i}$ is the external interference signals.

By this theory, the control method based on the inverse PI model can reduce the hysteresis, but it is difficult to eliminate the influence from external interference signals.

\subsubsection{PID hybrid control method based on the particle swarm optimization with cross and heredity function}

In order to eliminate the external interference and improve the control accuracy, a PID hybrid control method whose parameters are adjusted by particle swarm optimization [24-26], with cross and heredity function is proposed for the sake of high control accuracy in this

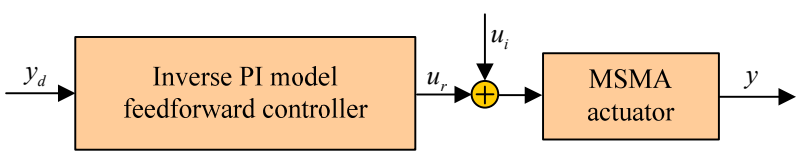

Fig. 4. Structure of open loop controller based on inverse PI model

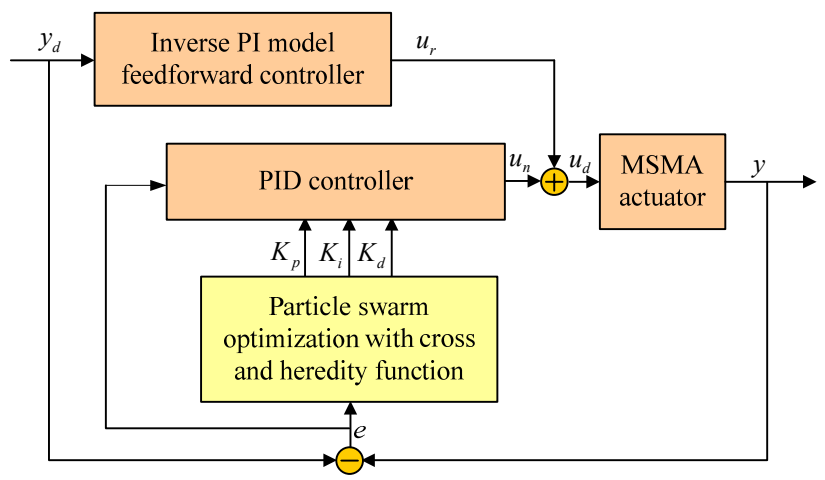

Fig. 5. Structure of hybrid control

section. The hybrid control structure is illustrated in Fig. 5, where $u_{d}$ is the actual input of the MSMA actuator, $u_{n}$ is the output of PID controller, $K_{p}, K_{i}$ and $K_{d}$ are the proportional gains, the integral gains and the derivative gains respectively.

The discretized PID controller can be expressed by

$$
y(k)=K_{p} e(k)+K_{i} \sum_{j=0}^{k} e(k)+K_{d}(e(k)-e(k-1)) / T_{s}
$$

where $y_{d}(k)$ is the input signal, and $y(k)$ is the output signal, $e(k)=y_{d}(k)-y(k)$ is the error between the output signal and the given signal, $T_{s}$ is the sampling time.

There are only three parameters to be identified in the PID controller, and the PSO is perfectly used for searching the proportional gains, the derivative gains and the integral gains. But, the classical PSO has a drawback that it is easy to fall into a local optimum. Consequently, the PSO with cross and heredity function is proposed in this paper. Its basic principle is that the genie crossover and heredity is added to the PSO, and the particle swarm with cross and heredity function has a certain mutation which is able to help the particle swarm jump out of the local optimum.

It is well-known that it is difficult to set the optimal PID parameters adjusted by the experience piece-try method. Hence, the PSO with cross and heredity function is used to obtain the optimal PID parameters.

Assuming the number of the entire particle swarm is $N$, each particle represents three parameters of the PID controller, and the entire particle swarm state can be shown by a $N \times 3$ matrix.

Each particle has four properties: the current position ( $\operatorname{positon}(i, j))$, the individual's best position ( pbest $(i)$ : the optimal solution found by the particle itself), speed $(\operatorname{speed}(i, j))$ and fitness $(\operatorname{fitness}[\operatorname{positon}(i, j)])$. When the searching time is equal to the maximum number of iterations (maxtime), the optimal solution is found. Where, $i$ is the $i$ th particle and $j$ is the its $j$ th parameter.

The basic idea of the parameters tuning of the PID controller based on the PSO with cross and heredity function is as follows. 
Step 1. The particle initialization

a) The $i$ th particle initialization

The position of all particles can be shown by a $N \times 3$ matrix

$$
\operatorname{position}(i,:)=\left[K_{p}, K_{i}, K_{d}\right], \quad(i=1,2, \cdots, N)
$$

where $\left[K_{p}, K_{i}, K_{d}\right]$ represents three parameters of the PID controller.

b) The initialization of the $i$ th particles' speed

The randn is a random matrix, and the $i$ th particles' speed is

$$
\operatorname{speed}(i, j)=\operatorname{randn}, \quad(i=1,2, \cdots, N ; j=1,2,3)
$$

c) The initialization of the individual's best position

The individual's best position is

$$
\operatorname{pbest}(i)=\operatorname{fitness}[\operatorname{position}(i,:)],(i=1,2, \cdots, N)
$$

d) The initialization of the fitness

$$
\text { fitness }[\operatorname{position}(i,:)]=1 / \sum_{t=1}^{n} \frac{1}{2}\left[y(t)-y_{d}(t)\right]^{2}
$$

where $(i=1,2, \cdots, N)$ is the fitness, $n$ is the total length of time, $y_{d}(k)$ and $y(t)$ are the object tracking signal and the actual output respectively.

e) The initialization of the best previous position among all the individuals

The highest fitness of all the particles is chosen as the best previous position among all the individuals

$$
\begin{gathered}
\text { gbest }=\max \{[\text { fitness }(\operatorname{position}(i,:))]\} \\
(i=1,2, \cdots, N)
\end{gathered}
$$

Step 2. According to the fitness, the particle is sorted. Half the particles with high fitness directly go into the next generation, and the other half, after crossover operation, go into the next generation.

One of the three parameters of the PID controller in the $(N / 2+i)$ th particle is selected to perform the crossover operation with the corresponding parameters of the $(N+1-i)$ th particle. It is shown in Fig. 6, position $_{N / 2+i}$ and position $_{N / 2-i}$ are the position matrix of the $(N / 2+i)$ th particle and the position matrix of the $(N+1-i)$ th particle respectively.

The $N / 2$ particles with high fitness serving as the next generation are selected from the $N / 2$ particles generating after the crossover operation and $N / 2$ particles generating before the crossover operation. The new selected $N / 2$ particles are obtained by the crossover operation between the first $N / 2$ particles without the crossover operation and the other $N / 2$ particles without the crossover operation.

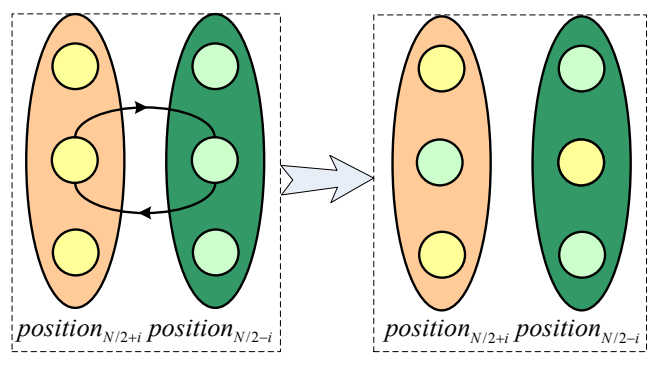

Fig. 6. Schematic diagram of the crossover operation

The next generational $N$ particles are composed of the $N / 2$ particles with high fitness and the new selected $N / 2$ particles.

Step 3. The speed and position of each particle are updated by (13) and (14).

$$
\begin{aligned}
& \operatorname{speed}(i)=w \times \operatorname{speed}(i) \\
& +c 1 \times \operatorname{rand} \times[\text { pbest }(i)-\operatorname{position}(i)] \\
& +c 2 \times \text { rand } \times[\text { gbest }-\operatorname{position}(i)] \\
& \quad(i=1,2, \cdots N) \\
& \operatorname{position}(i,:)=\operatorname{position}(i,:)+\operatorname{speed}(i,:)
\end{aligned}
$$

where rand is a random number, $w$ is the inertia coefficient, $c 1$ and $c 2$ are acceleration constants.

Step 4. The updates of the individual's best position and the best previous position among all the individuals.

The updates of the individual's best position: if the fitness of the updated position is higher than the previous position, $\operatorname{pbest}(i)=\operatorname{position}(i,:)$. Otherwise, $\operatorname{pbest}(i)$ is unchanged.

Step 5. Stopping condition: if the current iterations $G$ is equal to maxtime, then stop. Otherwise, set $G=G+1$ and go to step. 2 .

\section{Simulation Research}

\subsection{Simulation results of open loop controller of the MSMA actuator}

By the feedforward controller, the influence of the hysteresis nonlinearity from the MSMA actuator can be compensated. After the desired output signal passes through the inverse PI model, an inverse compensation signal is obtained, and it is shown in Fig. 7. The obtained inverse compensation signal can decrease the error between the desired output and the actual output of the MSMA actuator.

As the inverse compensation signal passes through the MSMA actuator, it can acquire that the actual output signal of the MSMA actuator is similar to the desired output signal. The simulation results of the open loop control method are shown in Fig. 8, 9 and 10. The tracking 


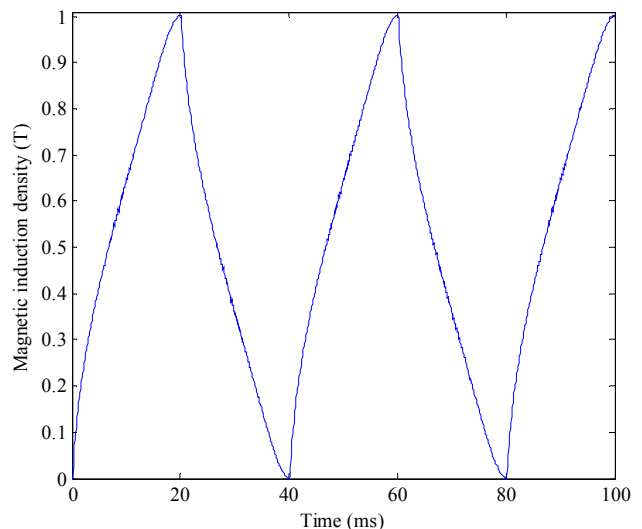

Fig. 7. Compensation signal obtained by the inverse PI model

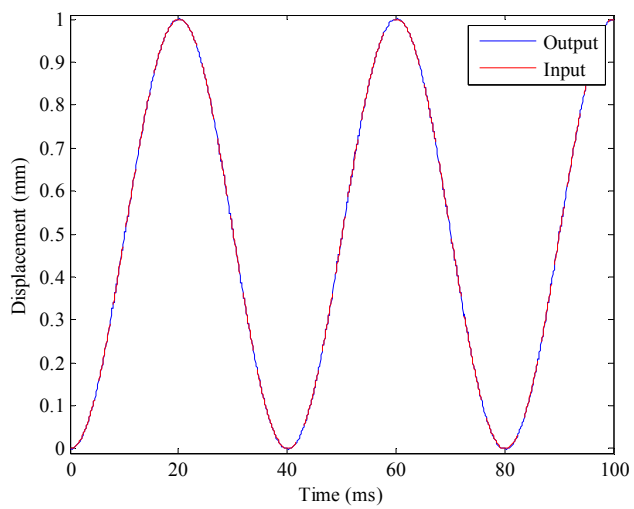

Fig. 8. Displacement tracking curve of the open loop control method

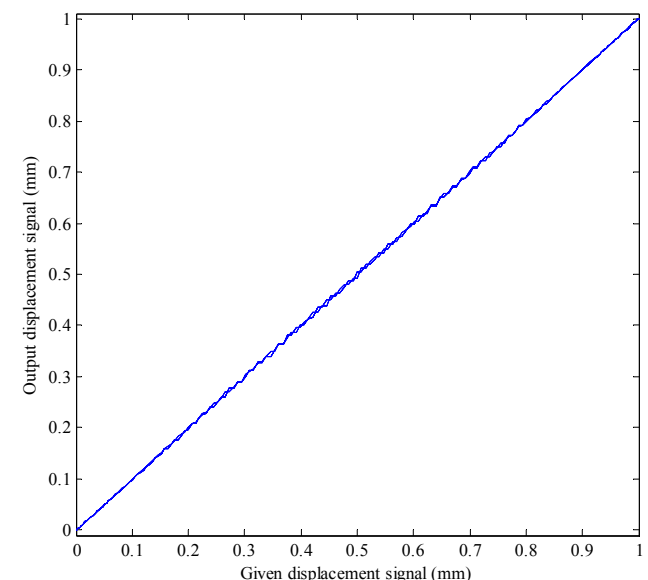

Fig. 9. Input-output relation curve of the open loop control method

displacement curve of the open loop control method is shown in Fig. 8. The red dotted line and the blue solid line represent the desired output signal of displacement and the actual output signal of displacement. The hysteresis curve and the tracking error curve of displacement of the open loop control method are shown in Fig. 9 and 10. The simulation results demonstrate that the error rate and the

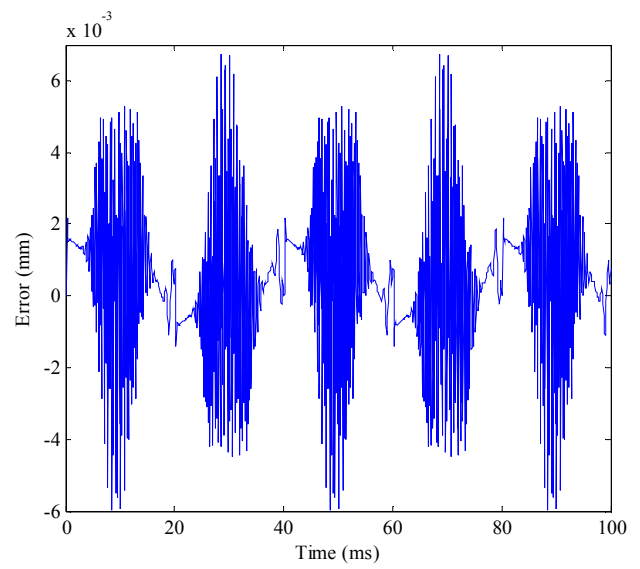

Fig. 10. Displacement tracking error curves of the open loop control method

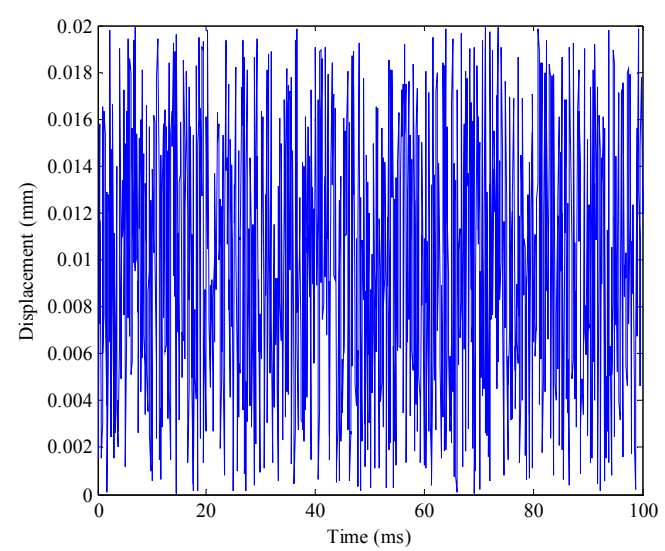

Fig. 11. Given external interference signals

mean square error of the open loop control method are $0.0067 \mathrm{~mm}$ and 0.0023 respectively, and it proves that the open loop control method can enhance the displacement tracking accuracy for the MSMA actuator.

In order to study the control effectiveness under external interference, a random data set $u_{i}$ that their maximum amplitude is $0.02 \mathrm{~mm}$ is chosen as the external interference signals in Fig. 11.

The simulation results of the open loop control method under the external interference signals are shown in Fig. 12 and 13. The tracking displacement of the open loop control method under the external interference signals is shown in Fig. 12. The red dotted line and the blue solid line represent the desired output signal of displacement and the actual output signal of displacement. The displacement tracking error curve of the open loop control method under the external interference signals is shown in Fig. 13. The simulation results demonstrate that the error rate and the mean square error of the open loop control method under the external interference signals are $3.29 \%$ and 0.0084 respectively, and it proves that the open loop control method cannot eliminate the influence of external interference signals. 


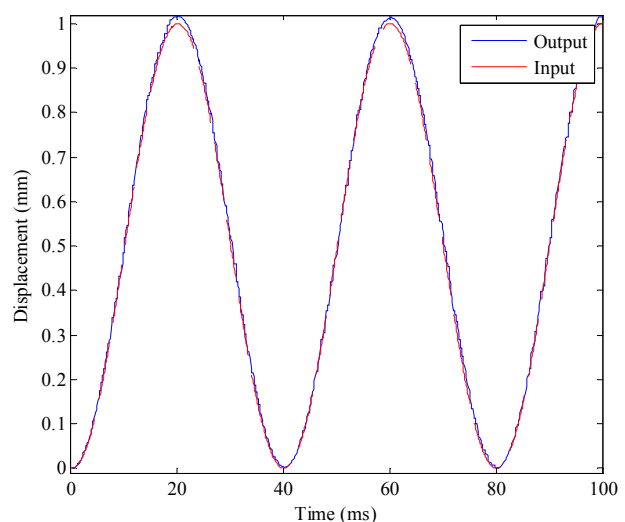

Fig. 12. Displacement tracking curve of the open loop control method under the external interference signals

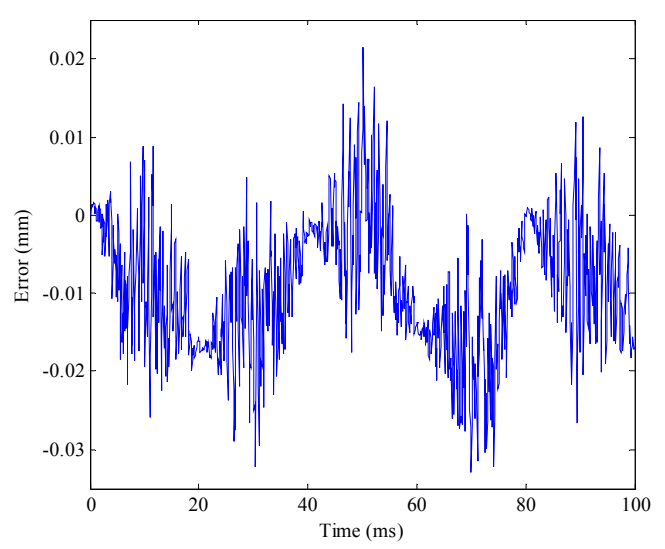

Fig. 13. Displacement tracking error curve of the open loop control method under the external interference signals

\subsection{Simulation results of the PID hybrid control method based on the PSO with cross and heredity function}

According to the experience piece-try PID parameters tuning method, PID parameters which can achieve the best performance are $K_{p}=0.01, K_{i}=0.001$ and $K_{d}=0.02$ respectively.

PSO is a new robust stochastic evolutionary computational algorithm based on the intelligence of swarms. One of the desirable merits of the PSO is that the obtained PID parameters can be used as the initial value of particle. The number of particles and the maximum number of the PSO iterations are $N=8$ and maxtime $=50$. The PID parameters which can be obtained by the PSO with cross and heredity function are $K_{p}=0.0161, K_{i}=0.00004$ and $K_{d}=0.0242$ respectively. Comparing with the PID parameters obtained by the experience piece-try method, the more accurate parameters of the PID controller can be obtained by using the PSO with cross and heredity function.

The simulation results of the PID hybrid control method based on the PSO with cross and heredity function are

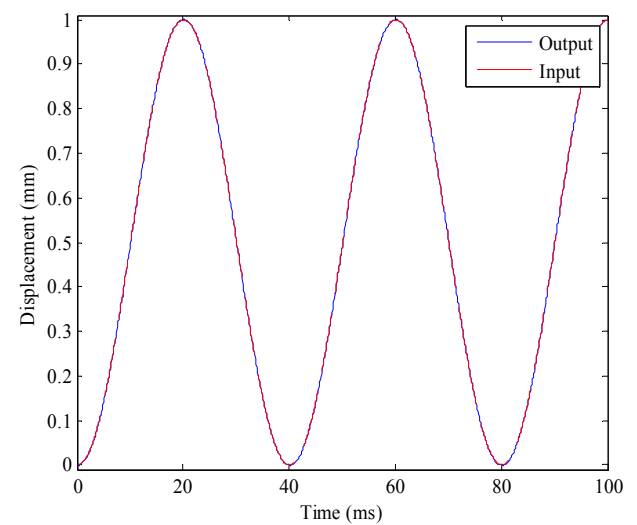

Fig. 14. Displacement tracking curve of the PID hybrid control method based on the PSO with cross and heredity function

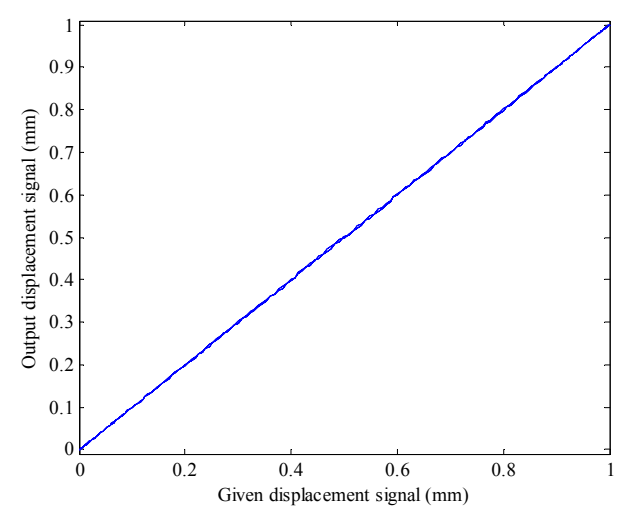

Fig. 15. Input-output relation curve of the PID hybrid control method based on the PSO with cross and heredity function

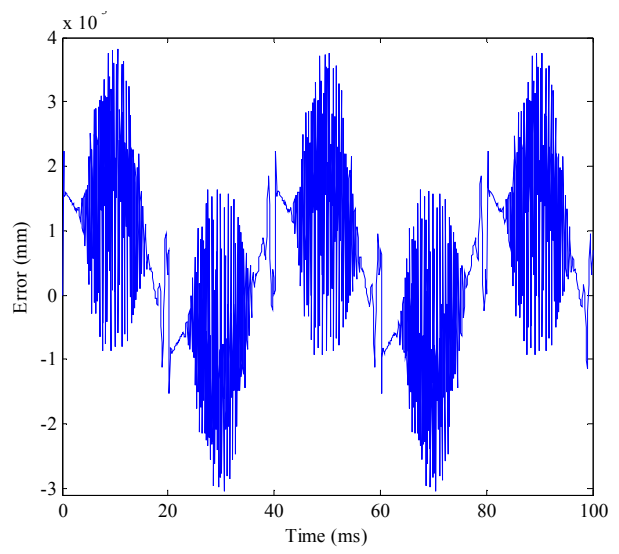

Fig. 16. Tracking displacement error curve of the PID hybrid control method based on the PSO with cross and heredity function

shown in Fig. 14, 15 and 16. Fig. 14 shows the tracking displacement effectiveness of the PID hybrid control method based on the PSO with cross and heredity function. The red dotted line and the blue solid line represent the desired output signal of displacement and the actual output 


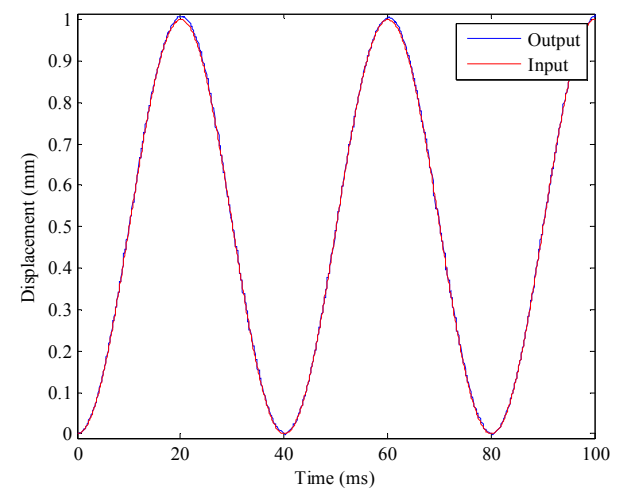

Fig. 17. Displacement tracking curve of the PID hybrid control method based on the PSO with cross and heredity function under the external interference signals

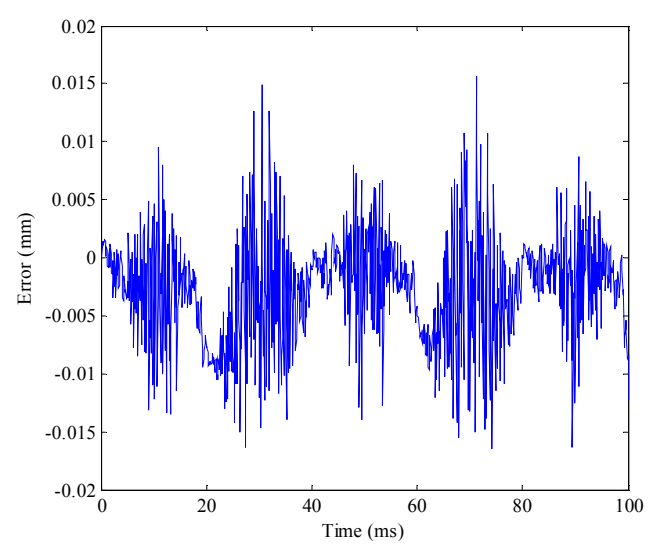

Fig. 18. Displacement tracking error curve of the PID hybrid control method based on the PSO with cross and heredity function under the external interference signals

signal of displacement. Fig. 15 shows the hysteresis curve of the PID hybrid control method based on the PSO with cross and heredity function. Fig. 16 shows the tracking displacement error curve of the PID hybrid control method based on the PSO with cross and heredity function. It can be seen from the simulation data that the error rate and the mean square error of the PID hybrid control method based on the PSO with cross and heredity function are $0.38 \%$ and 0.0014 respectively, and the control accuracy of PID hybrid control based on the PSO with cross and heredity function is improved from $0.67 \%$ (open loop control method) to $0.38 \%$. So the positioning accuracy obtained by the PID hybrid control method based on the PSO with cross and heredity function which has the advantage for controlling the MSMA actuator has been improved obviously.

The simulation results of the PID hybrid control method based on the PSO with cross and heredity function under the external interference signals are shown in Fig. 17 and 18. The tracking displacement effectiveness is shown in Fig. 17. The red dotted line and the blue solid line represent the desired output signal of displacement and the actual output signal of displacement. The displacement tracking error curve is shown in Fig. 18. The simulation results demonstrate that the error rate and the mean square error are $1.65 \%$ and 0.0046 respectively, and the control accuracy of the PID hybrid control method based on the PSO with cross and heredity function under the external interference signals is improved from $3.29 \%$ (open loop control method under the external interference signals) to $1.65 \%$. So it can prove that the PID hybrid control method based on the PSO with cross and heredity function can eliminate the external interference and improve the positioning accuracy for the MSMA actuator.

\section{Conclusion}

The inverse PI model has the same structure as the PI model, and the basic hysteresis operator of the inverse model (stop operator) can be directly calculated by the basic hysteresis operator of the PI model (play operator). By this theory, this paper proposes the inverse PI model of the hysteresis model of the MSMA actuator whose weights are identified by the RWLS algorithm. Subsequently, the established inverse model is adopted as the feedforward controller of the MSMA actuator.

As the feedforward controller is established, an open loop control method is used to improve the control accuracy of the MSMA actuator. To further improve the control accuracy, a PID hybrid control method which uses the inverse Prandtl-Ishlinskii model as a feedforward controller is proposed. In order to obtain the optimal PID parameters, a PSO method with cross and heredity function is designed. It can overcome this drawback that the PID parameters adjusted by the classical PSO drops into the local optimum. The simulation results demonstrate that the maximum tracking displacement error of the proposed PID hybrid control method is reduced from $0.67 \%$ in the open loop control method to $0.38 \%$. That is to say, it is improved by nearly half time in comparison to the control accuracy acquired by the open loop control method. Considering the influence of external interference disturbance, the maximum tracking displacement error of the proposed PID hybrid control method is reduced from $3.29 \%$ in the open loop control method to $1.65 \%$. It can be seen that the proposed PID hybrid control method can improve the antiinterference ability of the system and can achieve the highprecision positioning control of the MSMA actuator.

\section{Acknowledgements}

This research is partially supported by National Natural Science Foundation of China (Grant No.: 51105170), Program of Science and Technology Development Plan of Jilin province of China (Grant No.: 20140101062JC, 20140101082JC), and Program of Twelfth Five-Year 
Science and Technology Research Plan of Education Department of Jilin province of China (Grant No.: 2014B023).

\section{References}

[1] A. Sozinov, A. Likhachev and K. Ullakko, "Crystal Structures and Magnetic Anisotropy Properties of Ni-Mn-Ga Martensitic Phases with Giant MagneticField-Induced Strain," IEEE Transactions on Magnetics, vol.38, No.5, pp. 2814-2816, Sept. 2002.

[2] S. Murray, R. Hayashi, M. Marioni and S. Allen, "Magnetic and Mechanical Properties of FeNiCoTi and NiMnGa Magnetic Shape Memory Alloy," in Symposium on Smart Structures and Materials, Newport Beach, CA, USA, pp. 204-211, Mar. 1999.

[3] I. Suorsa, J. Tellinen, E. Pagounis, I. Aaltio and K. Ullakko, "Applications of Magnetic Shape Memory Actuators," in Proceeding of 8th International Conference on Actuator, Bremen, Germany, pp. 158-161, Jun. 2002.

[4] J. Tellinen, I. Suorsa and A. Jääskeläinen, "Basic Properties of Magnetic Shape Memory Actuators," in Proceeding of 8th International Conference on Actuator, Bremen, Germany, pp. 566-569, Jun. 2002.

[5] K. Ullakko, "Magnetically Controlled Shape Memory Alloys: A New Class of Actuator Materials," Journal of Materials Engineering and Performance, vol.5, No.3, pp. 405-409, Jun. 1996.

[6] K. Matsunaga, N. Niguchi and K. Hirata, "Study on Starting Performance of Ni-Mn-Ga Magnetic Shape Memory Alloy Linear Actuator," IEEE Transactions on Magnetics, vol.49, No.5, pp. 2225-2228, May 2013.

[7] M. Zhou, W. Gao and L. Gao, "Hysteresis Model of Magnetic Shape Memory Alloy Actuator," Journal of Jilin University(Engineering and Technology Edition), vol.42, No.3, pp. 714-718, May 2012.

[8] Y. Chen, J. Qiu, J. Palacios and E. Smith, "Tracking Control of Piezoelectric Stack Actuator Using Modified Prandtl-Ishlinskii," Journal of Intelligent Material Systems and Structires, vol.24, No.6, pp. 753-760, Apr. 2013.

[9] L. Riccardi, D. Naso, B. Turchiano and H. Janocha, "Adaptive Control of Positioning Systems with Hysteresis Based on Magnetic Shape Memory Alloys," IEEE Transactions on Control Systems Technology, vol.21, No.6, pp. 2011-2023, Jun. 2013.

[10] X. Zong, N. Zhang and P. Wang, "Study on Control Strategy of Nonlinear Systems with Hysteresis Inverse Model Feed-Forward Compensation Based on Preisach," in Advanced Materials Research, Wuhan, Hubei, China, pp. 261-264, Oct. 2013.

[11] O. Amor, L. Marc, K. Azeddine and A. Rachid, "PSO Based Parameter Identification of Colman-
Hodgdon Hysteresis Model of a Piezoelectric Actuator and PID Feedback Controller," Contemporary Engineering Sciences, vol.7, No4, pp. 179192, 2014.

[12] G. Wang, C. Guan, X. Zhang, H. Zhou and C. Rao, "Precision Control of Piezo-actuated Optical Deflector with Nonlinearity Correction Based on Hysteresis Model," Optics and Laser Technology, vol.57, pp. 26-31, Apr. 2014.

[13] L. Riccardi, D. Naso, H. Janocha and B. Turchiano, "A Precise Positioning Actuator Based on Feedback controlled Magnetic Shape Memory Alloys," Mechatronics, vol.22, No.5, pp. 568-576, Aug. 2012.

[14] G. Gu and L. Zhu, "High-speed tracking control of piezoelectric actuators using an ellipse-based hysteresis model," Review of Scientific Instruments, vol.81, No.8, pp. 085104, Aug. 2010.

[15] H. Sayyaadi and M. Zakerzadeh, "Position Control of Shape Memory Alloy Actuator Based on the Generalized Prandtl-Ishlinskii Inverse Model," Mechatronics, vol. 22, No. 7, pp. 945-957, Oct. 2012.

[16] D. Hughes and J. Wen, "Preisach Modeling of Piezoceramic and Shape Memory Alloy Hysteresis," Smart Materials and Structures, vol.6, No.3, pp. 287300, Jun. 1997.

[17] I. Mayergoyz and G. Friedman, "Generalized Preisach Model of Hysteresis," IEEE Transactions on Magnetics, vol.24, No.1, pp. 212-217, Jan. 1987.

[18] Y. Chen, J. Qiu and H. Sun, "A Hybrid Model of Prandtl-Ishlinskii Operator and Neural Network for Hysteresis Compensation in Piezoelectric Actuators," International Journal of Applied Electromagnetics and Mechanics, vol. 41, No. 3, pp. 335-347, Mar. 2013.

[19] L. Juhás, J. Maas and B. Borovac, "Parameter Identification and Hysteresis Compensation of Embedded Piezoelectric Stack Actuators," Mechatronics, vol. 21, No. 1, pp. 329-338, Feb. 2011.

[20] J. Yang and S. Lin, "Identification of Parametric Variations of Structures Based on Least Squares Estimation and Adaptive Tracking Technique," Journal of Engineering Mechanics, vol. 131, No. 3, pp. 290-298, Mar. 2005.

[21] G. Zhang, C. Zhang and K. Li, "Adaptive Identification and Inverse Control of Piezoelectric Actuators Based on PI Hysteresis Model," Nanotechnology and Precision Engineering, vol.11, No.1, pp. 85-89, Jan. 2013.

[22] P. Krejci and K. Kuhnen, "Inverse Control of Systems with Hysteresis and Creep," IEE ProceedingsControl Theory and Applications, vol.148, No.3, pp. 185-192, May 2001.

[23] M. Hadef and M. Mekideche, "Inverse problem methodology for parameter identification of a separately excited DC motor," Journal of Electrical Engineering and Technology, vol.4, No.3, pp. 365- 
369, Sept. 2009.

[24] T. Kim, B. Lee and H. Song, "Optimal capacitor placement considering voltage-stability margin with hybrid particle swarm optimization," Journal of Electrical Engineering and Technology, vol.6, No.6, pp. 786-792, Nov. 2011.

[25] R. Kuo and Y. Han, "A Hybrid of Genetic Algorithm and Particle Swarm Optimization for Solving Bilevel Linear Programming Problem - A Case Study on Supply Chain Model," Applied Mathematical Modeling, vol. 35 No. 8, pp. 3905-3917, Feb. 2011.

[26] Y. Chang, "Fitness sharing particle swarm optimization approach to FACTS installation for transmission system loadability enhancement," Journal of Electrical Engineering and Technology, vol. 8, No. 1, pp. 31-39, Jan. 2013.

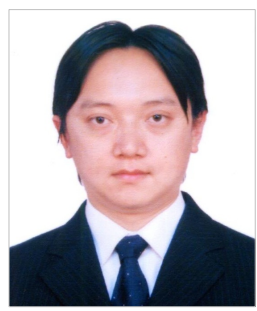

Miaolei Zhou. He received the B.S. and M.S. degrees in industrial electric automation from the Jilin Institute of Technology, China in 1997 and 2000, and the Ph.D. degree in control theory and control engineering from Jilin University, China, in 2004. Since 2000, he has worked in Jilin University, China. From 2006 to 2008, he was a postdoctoral researcher in the Tokyo University, Japan. Since 2014, he has been a Professor with the department of Control Science and Engineering, Jilin University, China. His research interests include micro/nano drive and control technology, nonlinear control theory.

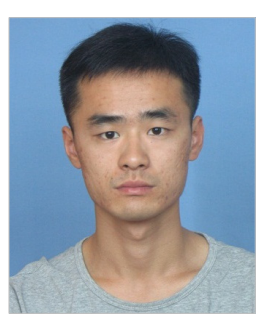

Rui Xu. He received the B.Sc. degree in Automation from Changchun University of Technology in 2014. Currently, he is a postgraduate student in College of Communication Engi-neering, Jilin University, China. His research interest is the hysteresis of magnetically controlled shape memory alloy actuators.

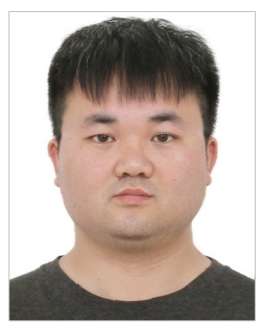

Qi Zhang. He received the B.Sc. degree in Automation from Heilongjiang University in 2012. He received the M.S. degree in College of Communication Engineering in Jilin University, China, in 2015. His research interest is the hysteresis of magnetic controlled shape memory alloy.

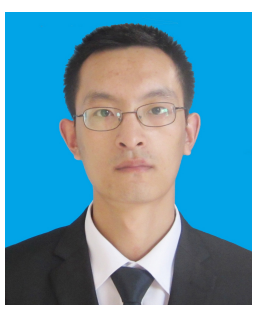

Ziying Wang. He received the B.Sc. degree in Communication Engineering from Jilin University in 2011. He received the M.S. degree in College of Communication Engineering in Jilin University, China, in 2014. His research interest is the hysteresis of magnetic controlled shape memory alloy.

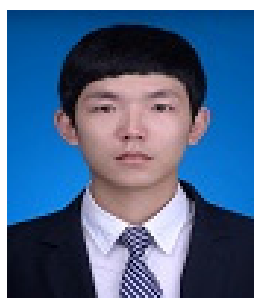

Yu Zhao. He received the B.Sc. degree in electrical engineering and automation from Changchun University of Science and Technology in 2013. Currently, he is a postgraduate student in College of Communication Engineering, Jilin University, China. His research interest is the hysteresis of piezoelectric actuators. 